\title{
Die AO auf dem DKOU 2016
}

\author{
Philip Wilbrandt, Michael J. Raschke
}

Auf dem diesjährigen Kongress der Deutschen Gesellschaft für Orthopädie und Unfallchirurgie (DKOU) war die AO mit insgesamt 13 wissenschaftlichen Sitzungen von AOTrauma, AOSpine, AOCMF und AORECON sowie dem AO-Forschungsinstitut (ARI), 5 öffentlichen Veranstaltungen am AO-Stand und sowie 3 Empfängen aktiv vertreten.

\section{Symposium der AOTrauma Deutschland „Renaissance vergessener Verfahren“}

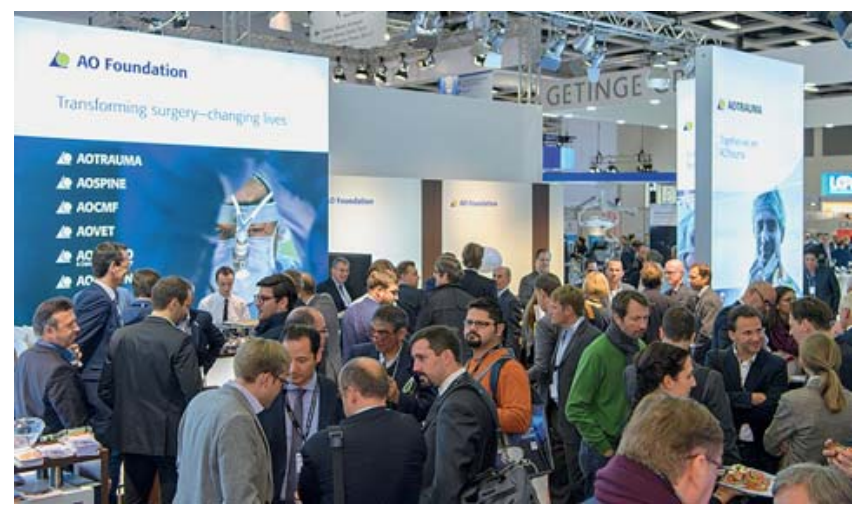

Abb. 1 Der Stand der $\mathrm{AO}$ auf dem DKOU. Foto: Jürgen Staiger, AO.

Am Mittwoch, 26.10.2016 fand die Sitzung „Zurück in die Zukunft: Renaissance vergessener Verfahren“" von der Deutschen AOTrauma mit 494 Teilnehmenden statt.

Marius Keel (Bern, CH) empfahl die Verwendung der Klingenplatte für bestimmte A3-Frakturen, insbesondere für Hochransanztraumata und junge Patienten und für Revisionen wie Nonunion bei pertrochantären Frakturen. Diese Expertise gehe leider zunehmend verloren und sollte daher geschult werden.

Michael Raschke (Münster) sah einen Bedarf für die Doppelplattenosteosynthese bei Revisionseingriffen (anteromediale/ventrale Platte), Überbrückung von „stress risern“ und periprothetischen Frakturen.

Hans Zwipp (Dresden) referierte über die Außenbandruptur, für die operative Strategien mit akuter Außenbandnaht in max. $2 \%$ notwendig sind, z. B. bei zusätzlicher osteochondraler Fraktur, offene Verletzung, manifestes oder drohendes Kompartmentsyndrom, Verletzung des Innenbands mit Luxatio pedis cum talo oder geschlossen irreponible knöcherne Bandausrisse.

OP-JOURNAL 2016; 32: 258-259

(c) Georg Thieme Verlag KG Stuttgart · New York DOI http://dx.doi.org/10.1055/s-0042-120726

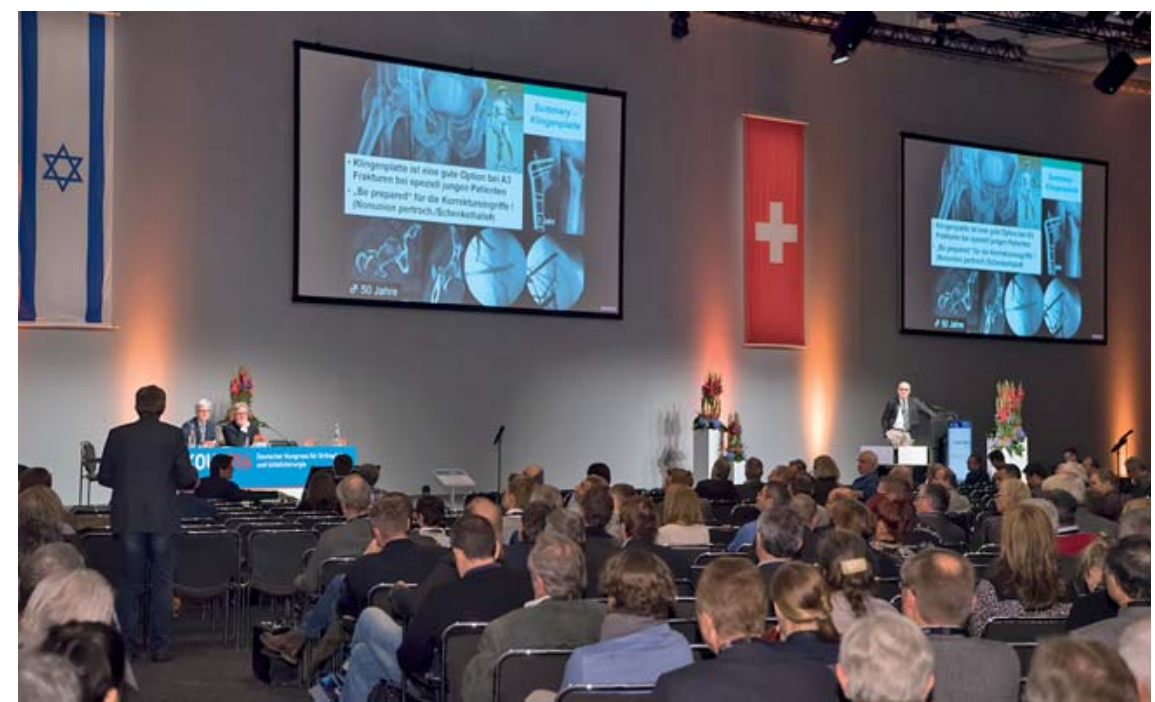

Abb. 2 Symposium der AOTrauma Deutschland. Foto: Jürgen Staiger, AO.

Daniel Rikli (Basel, $\mathrm{CH}$ ) verwendet die dorsale Platte beim distalen Radius noch bei dorsoulnarem Fragment/Impressionsfraktur (zentral), komplexen Frakturen mit karpaler Luxation und bestimmten frühen Korrekturen und Malunion vom dorsoulnaren Fragment.

Ingo Marzi (Frankfurt) empfahl die elastische Marknagelung des Unterarms im Kindesalter insbesondere für Frakturen beider Unterarmknochen, singuläre Radius- oder Ulnafrakturen und Grünholz-
Schaftfrakturen. Auch Korrekturosteotomien fehlverheilter Unterarmfrakturen können mit der elastischen Marknagelung stabilisiert werden. Grenzindikationen sind dislozierte Radiushalsfrakturen und Monteggia-Verletzungen. Die Plattenosteosynthese kann bei dislozierten dia-metaphysären Frakturen ohne Überschreitung der Wachstumsfuge v.a. bei jungen Adoleszenten angewandt werden. Für die elastische Marknagelung bei Erwachsenen bietet dieses Verfahren nicht genügend Stabilität. 


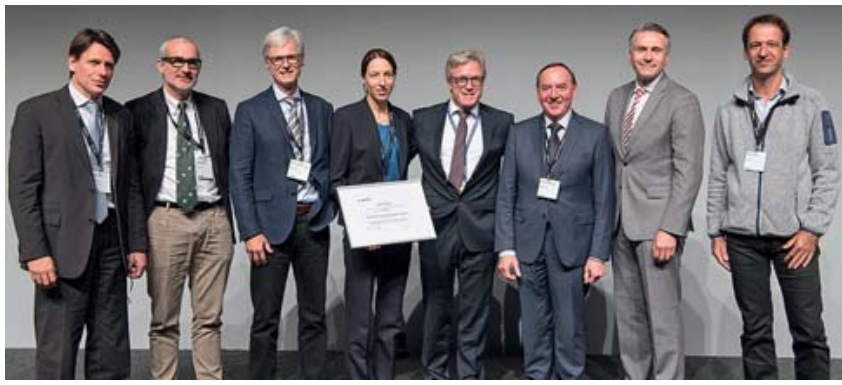

Abb. 3 Die Referenten und die gekürte Wissenschaftspreisträgerin. Foto: Jürgen Staiger, AO.

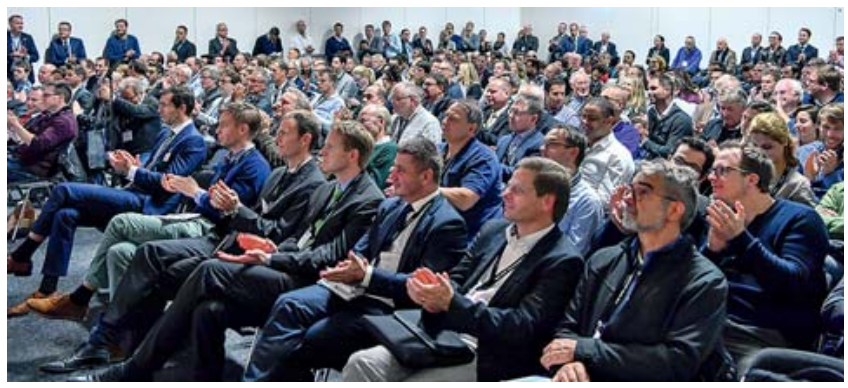

Abb. 4 Überfüllter Hörsaal. Foto: Jürgen Staiger, AO.

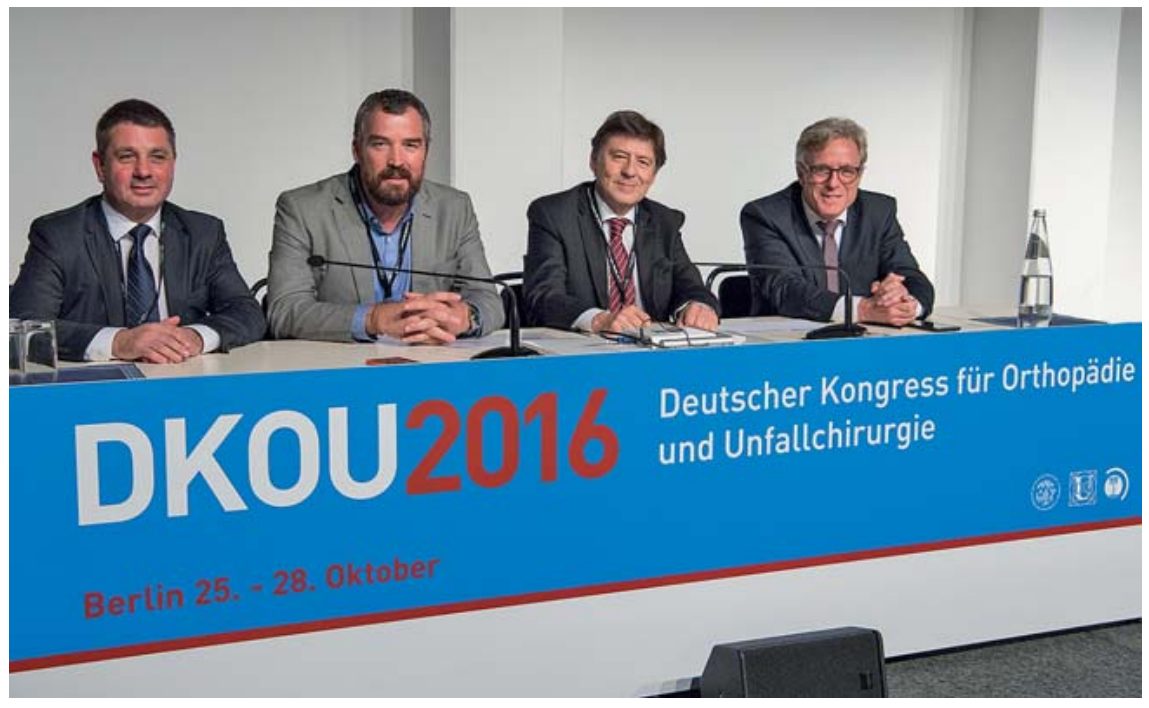

Abb. 5 Trampuz, Borens, Neubauer und Raschke. Foto: Jürgen Staiger, AO.

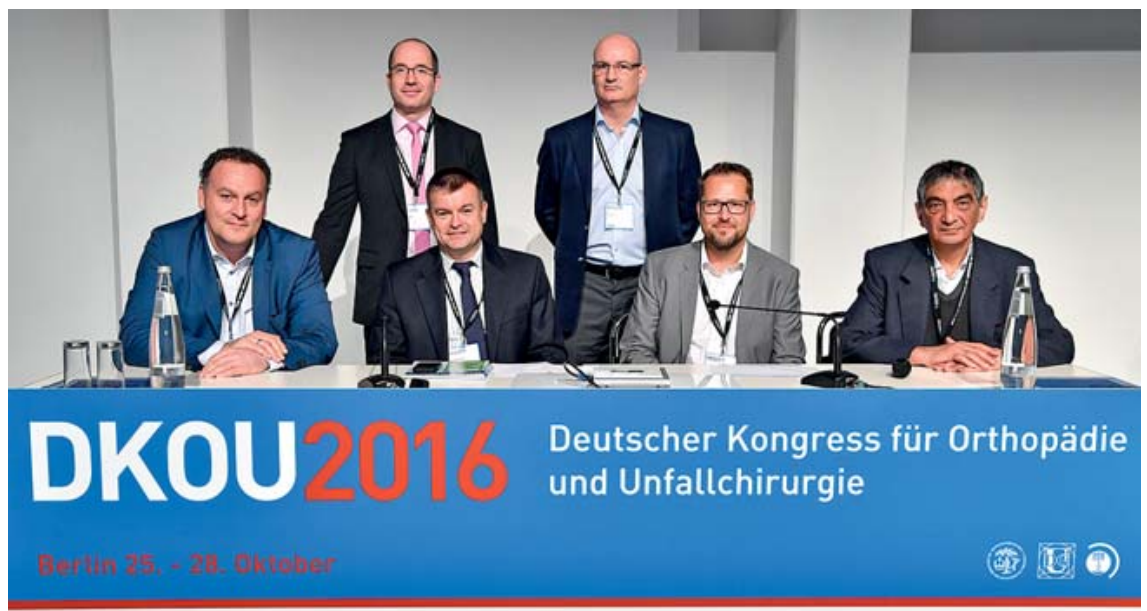

Abb. 6 Kandziora, Scholz, Schnake, Duff, Benneker und Öner. Foto: Jürgen Staiger, AO.

Reto Babst (Luzern, $\mathrm{CH}$ ) verwendet den Distraktor, um Ruhe und kontrollierte Stabilität ins Operationsfeld zu bringen. In Kombination mit einer Platte können selbst komplexe Frakturen minimalinva- siv und „indirekt“" versorgt werden. Der Distraktor dient hierbei als Repositionshilfe. Der Spreizer dient als Distraktionshilfe bei den kleineren Gelenken wie Talus und Ellbogen.
Christian Krettek (Hannover) stellte die Ergebnisse neuer randomisierter Studien bei dislozierten und instabilen Frakturen des proximalen Humerus vor, die fast identische Ergebnisse im Constant Score zwischen operativer und konservativer Behandlung zeigten, wobei die operative Gruppe eine deutlich erhöhte Komplikationsrate aufwies.

\section{Gemeinsames Symposium der AO Deutschland-Österreich-Schweiz (D-A-CH)}

Eine gemeinsame Veranstaltung der 3 AOTrauma-Sektionen von Deutschland, Österreich und Schweiz (D-A-CH) fand zum Thema Implantatinfektionen statt. 258 Teilnehmende im überfüllten Hörsaal verfolgten und diskutieren die 3 Vorträge „10 Fehler bei der...

- Behandlung eines Implantatinfekts

- Antibiotikabehandlung beim Implantatinfekt,

- Behandlung einer offenen Fraktur“

\section{AOSpine}

Die deutsche AOSpine bot die wissenschaftliche Sitzung „Controversies in spinal surgery - is therapy in Germany too aggressive?" an. Frank Kandziora, Klaus Schnake und Matti Scholz diskutierten mit John Duff (Lausanne, $\mathrm{CH}$ ), Lorin Benneker (Bern, $\mathrm{CH}$ ) und Fetullah Cumhur Öner (Utrecht, NL) die Behandlung von Berstungsfrakturen des thorakolumbalen Übergangs. Den Vorträgen über Zugänge und konservativ vs. operativ folgte eine 40-minütige Falldiskussion, an der die 143 Teilnehmenden sich rege beteiligten.

\section{Philip Wilbrandt}

Rechlin

\section{Prof. Dr. Michael J. Raschke}

Münster 